\title{
SUPERVIVENCIA URBANA UNA PROPUESTA PARA LA EDUCACIÓN INTEGRAL
}

\section{Autores}

VICENTE JAVIER VALLS MARTINEZ EMPRESA DE ANIMACIÓN AEIOU

SAUL VICIANO SELMA

EMPRESA DE ANIMACIÓN AEIOU
ROBERTO J. GARCÍA ANTOLÍN

PROFESOR DELA UNIVERSITAT JAUME I DE CASTELLÓN

\section{Introducción}

Zapatos de piel, con suela de...., y de marca tal, pantalones modelo...., con bolsillos estilo.... y cremalleras en...., impermeable de ... y con ..., bolsa de... para llevar..., localizador espacial de...., analizador del consumo de .... todo esto y mas es totalmente necesario para que vayas a....,

Hoy en día no se nos ocurre a nadie realizar ninguna actividad si no vamos bien equipados, si no tenemos todo lo que consideramos imprescindible y básico. Pero, lo imprescindible y básico ¿qué es? ¿lo último en tecnología y diseño? ¿lo último en marcas y moda?...

Aquella idea de la actividad que debía adaptarse al individuo, a pasado a ser la del individuo que necesita un gran número de cosas, imprescindibles, para hacer la actividad. "iCómo no voy a tener esto!, para una vez que lo hago, lo hago en condiciones", faltaría tal vez añadir "y si no ¿qué dirán?".

Estamos llenando nuestra practica de tiempo libre de instrumentos a los cuales ponemos la etiqueta de necesarios, y además hemos considerado que como esos instrumentos los lleva uno mismo, la práctica de tiempo libre es mía, individual. Olivera (1995) comenta que en la sociedad moderna hay un "aumento de tendencia de las prácticas deportivas hacia las prácticas individuales". Estudios recientes (García Montes, Ruiz Juan y Hernández Rodríguez, 2001) confirman esta tendencia, Podríamos decir que la práctica colectiva esta constituida por un conjunto de prácticas individuales.

Lo que proponemos es jugar con los demás y no contra los demás, conjugar la relación entre diversión, juego y creatividad (López Quintas, 2000: 85), recuperar valores educativos para mejorar la comunicación social, estimular la cooperación, el espíritu de equipo, la solidaridad, respeto a los compañeros...
La actividad se desarrollará en el contexto urbano, en el entorno inmediato que nos rodea, y dentro de este espacio los participantes deberán ir superando pruebas, en ella se podrán experimentar las ventajas del grupo a la hora de conseguir los objetivos propuestos, han de trabajar en equipo, utilizar adecuadamente los recursos con que cuentan, buscar la solución mas adecuada para superar el problema planteado en función del material que disponen y de las posibilidades del grupo, saber hablar, escuchar y decidir dentro del grupo, han de conseguir sobrevivir.

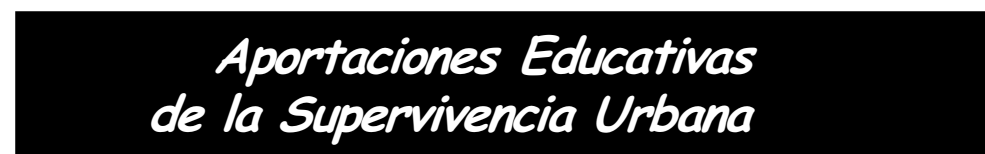

"Supervivencia es una ciencia, un deporte, un acertijo constante, según se la considere, pudiendo ser mucho más emocionante que una novela negra, ya que de aquí es a uno mismo a quien se le exige. Uno mismo debe resolver los problemas, y no el comisario de policía. Somos nosotros mismos quienes debemos ser astutos y no el guionista. Nosotros quienes tenemos que salir de los problemas, ya sea mediante trucos o a base de golpes. iUn campo de actividad verdaderamente fascinante!" (Nehberg, 1987).

¿Cabe éste tipo de práctica en nuestro entorno urbano más cercano?

La primera aproximación de las actividades de naturaleza a la ciudad, la encontramos en los recorridos de orientación que García Montes (1992) y García Montes y Hernández Rodríguez (1998: 56) nos describen, como necesidad de desarrollar las actividades de ocio y tiem- 
po libre en las urbes ya que "los problemas generados por el ritmo de vida que imponen las estructuras urbanas al día a día de sus ciudadanos, hacen cada vez más imperiosa la necesidad de desarrollar programas de ocio colectivo".

La propuesta que aquí hacemos, a la que denominamos "Supervivencia Urbana", pretende educar en el tiempo libre con la intención de aportar elementos para favorecer la transformación de la sociedad actual en la que "se oponen dos corrientes: por un lado el bienestar material y tecnológico, la riqueza de sus actividades y de sus recursos, y la intensidad de sus medios de comunicación; por otro lado, la superpoblación, las desigualdades sociales, la existencia artificial, la incomunicación, la ruptura con lo natural" (García Montes y Hernández Rodríguez, 1998: 55).

A través de las actividades diseñadas para la "Supervivencia Urbana" se desarrollan una serie de características que hacen de este gran juego no sólo una manera más de pasar un rato divertido, sino un instrumento a través del cual, entre otros aspectos. se potencia la educación en valores, se facilita la participación de todos los componentes del grupo, se posibilita la realización y vivencia de diferentes experiencias motrices y relacionales, se permite la transición y puesta en práctica de las sensaciones que se experimentan en las actividades de aventura y riesgo y se ayuda a la concienciación del grupo sobre las necesidades de integración de las personas con discapacidades físicas y sensoriales. A continuación pasamos a exponer, con más profundidad, cada uno de estos aspectos.

Potencia la educación en valores de entre los cuales destacaremos los siguientes:

\section{Igualdad de oportunidades para ambos sexos}

Las actividades que se proponen en el juego se basan más en actividades intelectuales, coordinativas y de toma de decisiones que en actividades donde prevalece el factor físico, lo que favorece la participación de los dos sexos por igual. También el intercambio constante de roles dentro del juego, permite que ambos sexos tengan la misma importancia en la consecución de los objetivos del grupo. Así pues, destacan las actividades cualitativas sobre las cuantitativas.

\section{Cooperación}

Nos encontramos con que la participación en este juego se realiza en pequeños grupos donde el éxito personal pasa por que exista un grado de cohesión grupal alto. Prevalece el grupo sobre la persona. Así mismo, cumple los tres criterios que todo juego cooperativo debe tener:

\section{- Sincronía Grupal \\ - Participación \\ - Diversión}

\section{Educación para la $\mathrm{Paz}$}

Respeto, responsabilidad, integración y democracia se desarrollan y transmiten a través de las actividades que en este juego se pueden realizar. La resolución de problemas continuos, hace que el grupo se enfrente continuamente a diferentes conflictos que les generan el propio objetivo de la actividad, el material a utilizar, el método a seguir, etc..., en los cuales el diálogo toma un importante protagonismo

\section{Respeto al medioambiente}

No sólo con la utilización de materiales de desecho para la realización de todas las actividades estamos educando para la conservación del medio ambiente, sino que además la economización de recursos es clave para el éxito de todas las acciones a realizar por el grupo, por lo que el reciclaje y la reutilización del material en cada actividad toman un papel muy importante dentro del juego.

Facilita la participación de todos los integrantes del grupo, ya que la dinámica del juego obliga a distribuirse en pequeños grupos, por lo que la participación de cada miembro del grupo se hace a su vez muy activa.

Además la tipología de las actividades realizadas invita a la participación de todo el grupo ya que en ellos se desarrollarán destrezas muy diferentes (coordinación, equilibrios, inteligencia, fuerza, velocidad, habilidad, orientación espacial, etc...), lo que favorece mucho la desaparición del rol que en muchos otros juegos es clave. Nos referimos al líder físicamente fuerte que realiza todas las pruebas y que eclipsa las habilidades y virtudes de sus compañeros de grupo.

Posibilita la realización y vivencia de diferentes experiencias motrices y relacionales gracias tanto a la variedad de actividades como a la diversidad de contenidos que en cada una de ellas se trabaja.

Permite la transmisión y puesta en práctica de las sensaciones que se experimentan en las actividades de aventura y riesgo, pero esta vez, trasladadas a nuestro entorno propio, al ámbito urbano, al día a día que vivimos en la ciudad.

Ayuda a la concienciación del grupo, de las necesidades de integración de las personas con discapacidades físicas y sensoriales. Como veremos a lo largo del juego el dinamizador va poniendo ciertos impedimentos con los cuales el grupo tiene que ir superando pruebas.

\section{Desarrollo del proceso del juego}

La "Supervivencia Urbana" podríamos englobarla como una actividad dentro de los Grandes Juegos, así como dinámicas grupales y juegos de rol. Está compuesta por diferentes variables y elementos que forman parte esencial del desarrollo del juego.

A continuación vamos a analizar el proceso de desarrollo del mismo (gráfico 1) así como todos sus elementos: 


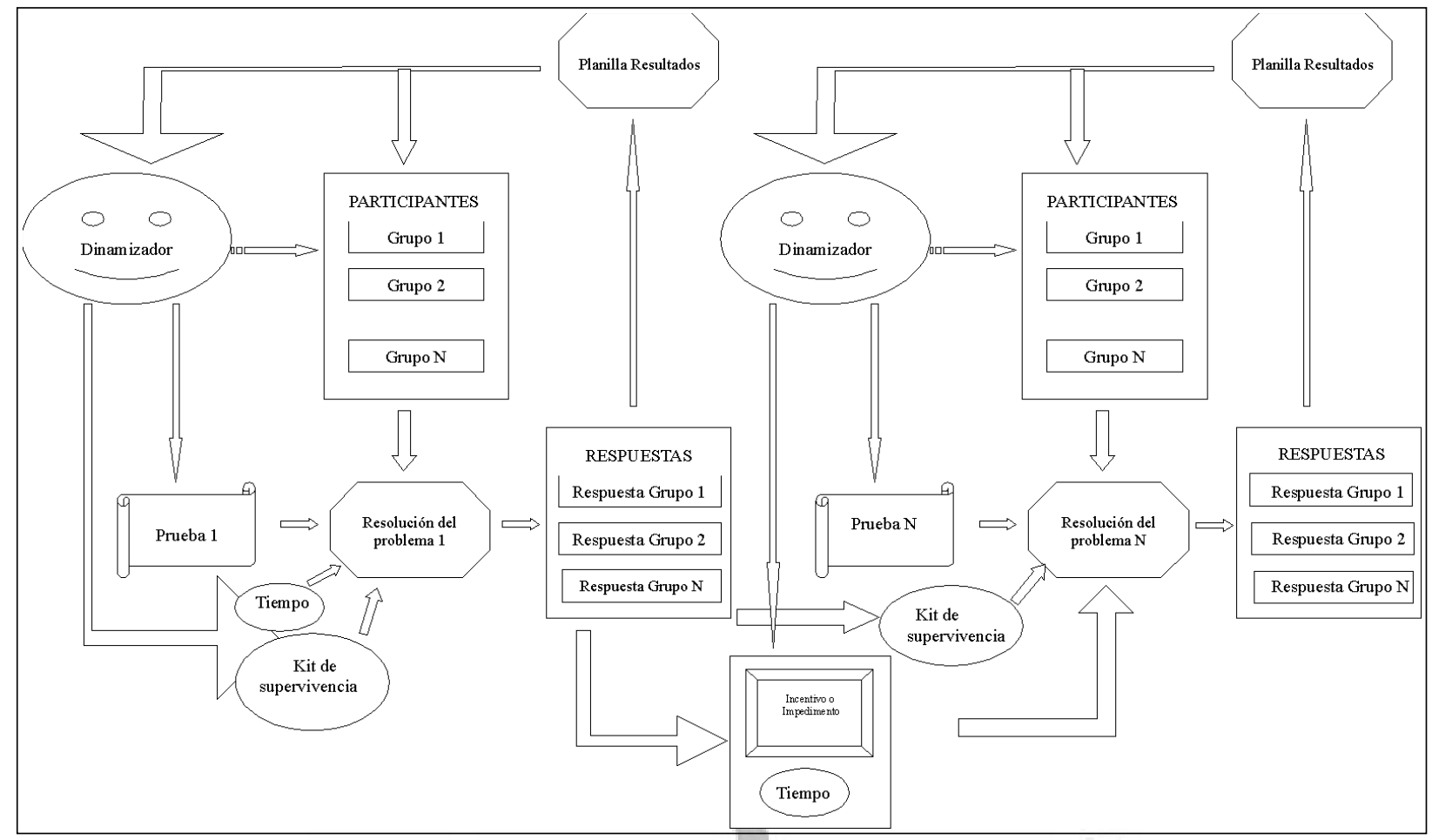

Gráfico 1. Proceso de desarrollo del juego "Supervivencia Urbana"

\section{LA DINÁMICA}

El juego comienza cuando el dinamizador, después de haber distribuido los participantes en grupos, reparte el kit de supervivencia compuesto, como veremos más adelante, por una bolsa con los materiales justos y necesarios para sobrevivir durante todo el juego. A continuación propone la primera prueba a hacer en un tiempo determinado por él mismo. Cada grupo tiene que buscar una solución que luego reflejará en la planilla de resultados. Dependiendo de si el resultado es satisfactorio o no, el dinamizador, para la siguiente prueba, dará un incentivo o pondrá un impedimento a cada grupo. Dentro de la práctica cada grupo no juega contra los demás sino que juega por lograr unos objetivos para el grupo, por conseguir su supervivencia.

\section{EL DINAMIZADOR}

El dinamizador es pieza clave para el buen desarrollo del juego, por lo que debe tener muy claro cuáles son sus funciones dentro del juego, así como tener muy bien definidas todas las variables que intervienen en él (las actividades, materiales, consignas, etc.).

Así el dinamizador deberá:

\section{Antes del juego}

- Ser consciente del conocimiento de los componentes del grupo, de forma que tenga presente las virtudes y defectos de cada uno de ellos.

- Diseñar y elegir las diferentes actividades del juego en función del grupo y de los objetivos que se quieran conseguir, así como adaptarlas al espacio y materiales disponibles.

- Valorar qué información va a dar en cada momento y cómo la va a dar.
- Establecer un criterio claro de superación de pruebas, de manera que se diera el incentivo o el impedimento por igual a todos los grupos de participantes.

- Elegir el material que formará parte del kit de supervivencia así como otros materiales que él necesite para preparar las actividades.

- Prever las diferentes respuestas y soluciones a un mismo problema que puedan dar cada uno de los grupos participantes.

\section{Durante el juego}

- Presentar las pruebas con la información que previamente se ha establecido como necesaria.

- Dar feed-backs positivos a todas las respuestas que se den y que ayuden al logro de los objetivos propuestos, sobre todo aquellas propuestas creativas, en la que haya participado y consensuado todo el grupo, con economización de recursos.

- Reconducir aquellas propuestas individuales.

- No dar ninguna solución a los problemas.

- Supervisar los resultados de las pruebas y dar el incentivo o el impedimento correspondiente previamente establecido.

- Controlar el tiempo de duración de cada una de las pruebas y de todo el juego en general.

\section{Al finalizar el juego}

- Realizar una valoración y reflexión con todo el grupo de cada una de las respuestas que se han dado en cada una de las pruebas resaltando la importancia en este tipo de práctica de:

- El trabajo en equipo

- La economía de recursos 
- La existencia de diferentes soluciones válidas para un mismo problema

- Ser conscientes de la influencia del estrés y las situaciones límite en la superación de las pruebas

- Valorar las aportaciones de cada individuo dentro del grupo para la superación de cada una de las pruebas

- Satisfacer las necesidades básicas del grupo para la consecución de un objetivo, antes que los caprichos individuales.

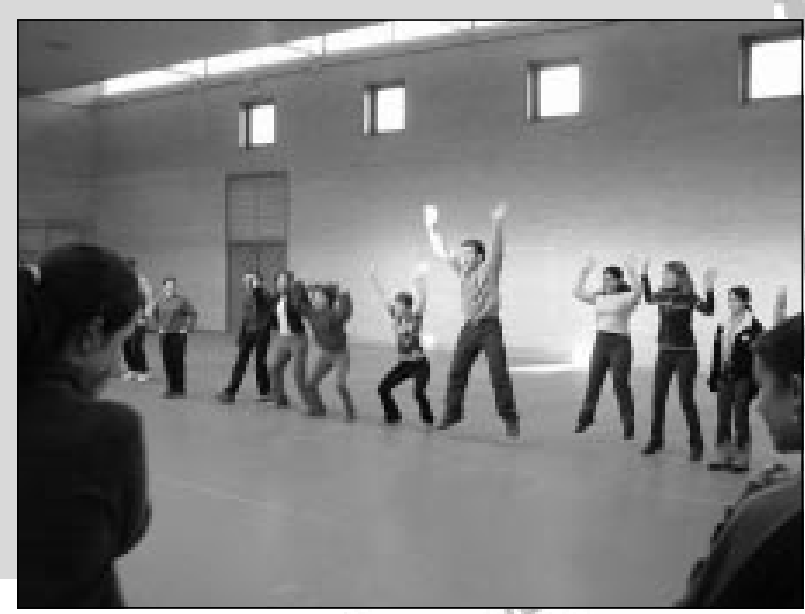

\section{LAS PRUEBAS}

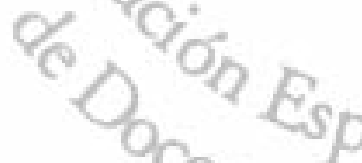

Las pruebas elegidas deben diseñarse para llevarnos a la resolución de un problema, a través del cual, podamos transmitir aquellos valores que nos ayuden a lograr los objetivos propuestos.

Las actividades que se diseñen deben estar encaminadas a las diferentes problemáticas que se puedan plantear, teniendo en cuenta:

- Los diferentes usos del material dentro de una pruebay dentro de todo juego así como la posibilidad de el reciclaje y la reutilización del mismo.

- Deben provocar el conflicto en la toma de decisiones del grupo.

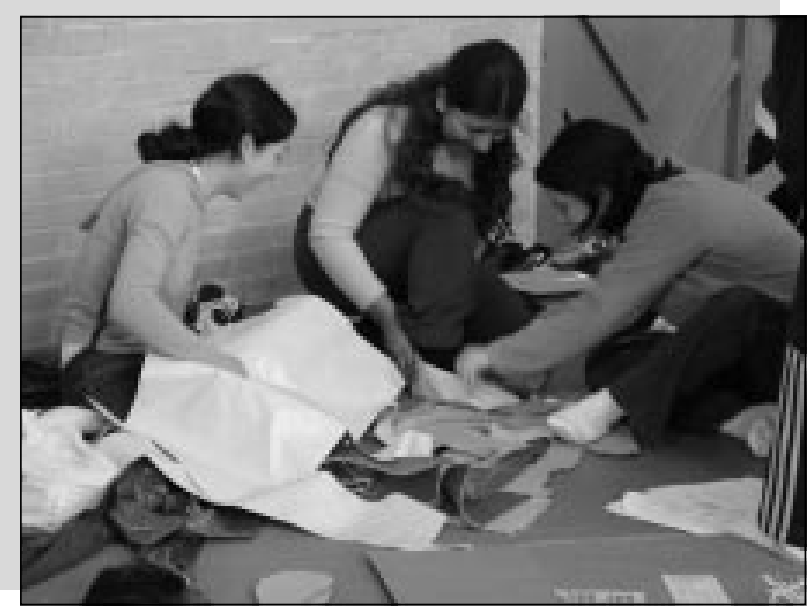

- El tiempo de duración de la prueba así como el tiempo total que disponemos para todo el juego.

- El espacio en el cuál se va a desarrollar el juego.

- Los contenidos que se quieren trabajar.

- La acumulación de impedimentos.

- Tienen que tener siempre una posibilidad de éxito.

- Puesto que estamos hablando de supervivencia, en este caso urbana, debemos transmitir contenidos referentes a actividades en la naturaleza como orientación, escalada, rastreo, etc.

Por otra parte y atendiendo al tipo de resultado las pruebas podrán ser:

- Con resolución objetiva, en la que la toma importancia el resultado y no el proceso, aunque el proceso puede influir en las resoluciones de pruebas posteriores.

- Con resolución subjetiva donde todas las soluciones son válidas siempre que el proceso hasta llegar a ella sea coherente con el objetivo perseguido.

\section{EL MATERIAL}

Dentro de los objetivos del juego, nos encontramos con transmisión de valores como la economía de recursos, el reciclaje y la reutilización, los cuales debemos tener en cuenta cuando vayamos a elegir el material a utilizar en el juego. Así pues elegiremos un material que sea:

- Fácil de adquirir

\section{- Económico}

- Propio de la vida cotidiana

- Con posibilidad de reciclaje y reutilización

- Fácil de utilizar

- Seguro y que esté dentro de las condiciones mínimas para su uso en actividades recreativas

El material que utilizaremos será, básicamente, no convencional y lo clasificaremos atendiendo a la estructura propuesta por Jardi y Pinyol (1990), que Ruiz Juan y García Montes (2001), analizan en profundidad. Así jugaremos principalmente con:

- Material de desecho de utilización inmediata: se puede utilizar sin ningún tipo de modificación (tubos de cartón, cajas, retales, etc...)

- Material de usos varios ajenos al ámbito de las actividades físico deportivas, fácilmente manipulable y muy polivalente que podemos adquirir en mercerías, ferreterías, todo a 1 euro... (cuerda, precinto, tijeras...)

- El entorno en el que nos encontremos no sólo estructuras urbanas sino también del medio natural (bancos, árboles, paredes, etc...)

El material utilizado cumplirá dos claras funciones dentro de nuestro juego (Ruiz Juan y García Montes, 2001):

- Función estructuradora o de transformación: los materiales son estructuradores o transformadores 
del espacio, alteran las referencias, tanto por su movilidad, como generando espacios de acción y aventura. Como veremos en la experiencia expuesta a continuación, durante el juego se van construyendo diversas zonas de juego con el material dado, de forma que el espacio en el cual se desarrolló la "Supervivencia Urbana" (polideportivo de un centro escolar, en el ejemplo que expondremos) quedó totalmente transformado.

- Función de inducción: "la información que producen los materiales, las relaciones perceptivas que generan, entre los participantes y el entorno, y su predisposición lúdica, provocan respuestas motoras diversas, animando los procesos imaginativos, estimulando determinados emocionales e incitando a la creatividad"(Ruiz Juan y García Montes, 2001: 178).

Como podemos observar el material nos ayudará a conseguir los objetivos que nos proponemos (creatividad, diferentes soluciones a un mismo problema, etc.) por lo que la elección de los componentes del kit de supervivencia que vamos a utilizar es muy importante dentro del juego.

Para el cumplimiento de los fines educativos propuestos, es necesario que la elección del material que vamos a proporcionar a los participantes sea la adecuada, por lo que la cantidad de material a dar será la justa de forma que los participantes aprendan a economizar y reutilizar el material, y a la vez el material nos debe posibilitar la realización y superación de todas las pruebas.

Este conjunto de material lo llamaremos el "Kit de Supervivencia", nombre que adoptamos del equipo de supervivencia dentro de esta actividad, en la naturaleza.

El "Kit de Supervivencia" será otra pieza clave dentro del juego, ya que dependiendo del uso que hagan de él, las pruebas se podrán superar con menor o mayor dificultad. Dependerá del dinamizador tanto la elección del "Kit de Supervivencia" como la modificación del mismo durante el juego (incentivo o impedimento) siempre que lo haya planificado con anterioridad.

\section{EL ESPACIO}

El juego que proponemos esta diseñado, como su propio nombre indica "Supervivencia Urbana", para ser desarrollado en espacios urbanos cuyas características más importantes son, que sean abiertos, con diferentes elementos físicos que lo compongan (farolas, bancos, fuentes, etc..), con un entorno con medio natural (plantas, árboles, etc...), con espacios lúdicos (estructuras y juegos infantiles que puedan existir como mobiliario urbano), etc. En definitiva necesitamos espacios como parques, plazas, centros urbanos, paseos, espacios polideportivos de calle, donde se pueda albergar un gran número de participantes, donde no exista circulación de vehículos y además nos permita diseñar y realizar una variedad amplia de actividades.

Debemos aprovechar los núcleos urbanos como "espacios para descubrir, siendo ricos tanto por la variedad, multiplicidad y combinaciones de sus estructuras físicas, como por el uso social que de ellas se hace" (García Montes y otros, 2000: 11).
Esto no quiere decir que no se pueda jugar a "Supervivencia Urbana" en otro tipo de espacios o instalaciones. Como veremos en el caso práctico, el juego se desarrolla en un instituto de secundaria donde se utilizaron espacios cerrados (polideportivo, pasillos y vestuarios) y espacios abiertos (zona ajardinada, pista polideportiva y parking). En este caso lo que se hizo fue diseñar las pruebas teniendo en cuenta los elementos que formaban cada uno de los espacios de que disponíamos para realizar el juego.

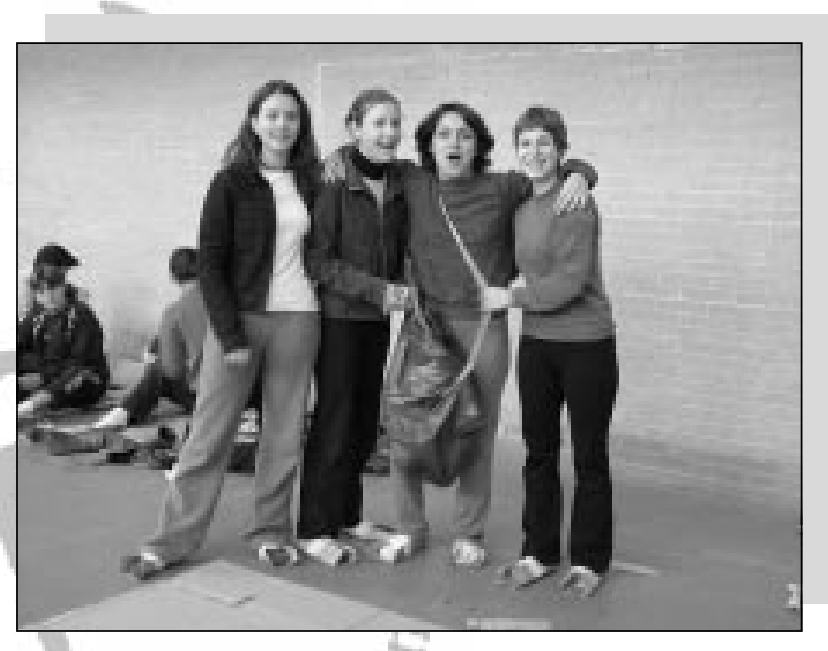

En otra ocasión, realizamos el juego en Peñíscola durante una escuela de verano, y se utilizó un polideportivo y el centro histórico de la ciudad como espacios base para la realización de todas las pruebas de "Supervivencia Urbana".

Así pues debemos buscar la utilización de espacios urbano que nos faciliten "sus posibles aprovechamientos no convencionales, que permiten el enriquecimiento personal al tiempo que ayudarán a la apropiación afectiva de la ciudad como conjunto" (García Montes y otros, 2000: 11).

\section{EL TIEMPO}

El tiempo va a ser pieza clave para la generación del estrés que dificultará la resolución de los problemas planteados. Así pues, lo entenderemos como agente externo que va a condicionar la práctica, de manera que en cada prueba se ha de fijar un tiempo tal, que permita el éxito de los participantes, pero ese tiempo no puede ser excesivo, ni tampoco muy escaso, ya que puede provocar una pérdida de interés en el desarrollo de la prueba $y$ en definitiva de todo el juego.

Por ello ante pruebas fáciles tendremos que dar poco tiempo de resolución y ante pruebas más dificultosas tendremos que ampliar más el tiempo para superarlas.

\section{EL INCENTIVO Y EL IMPEDIMENTO}

Este es otro agente externo que condicionará la práctica, provocando sorpresa e interés por la misma. Podemos entender el incentivo y el impedimento de tres formas diferentes:

- Respecto al material: dando o quitando material para la realización de la siguiente prueba. 
- Respecto al tiempo: aumentando o disminuyendo, al grupo, el tiempo de resolución de la prueba siguiente.

- Respecto al grupo: donde podemos modificar las características físicas o sensoriales de los componentes del grupo.

Este agente, puede aparecer de formas diferentes durante el juego. Algunas propuestas serían:

- Los impedimentos o incentivos son dados por el dinamizador según los criterios de superación de prueba que ha establecido con anterioridad. Esto le va a permitir regular y reconducir el juego hacia propuestas y dinámicas que favorezcan la consecución de los objetivos propuestos.

- Los impedimentos o incentivos pueden ser adjudicados al azar, utilizando un dado, una lista, etc.., donde son los propios participantes los que eligen, de forma que cabe la posibilidad que les facilite o dificulte la resolución de las pruebas siguientes. Aquí las decisiones del grupo toman gran importancia en el desarrollo de las pruebas.

\section{LA PLANILLA DE RESULTADOS}

Pondremos a disposición de todos los grupos de participantes una planilla de control donde cada grupo apuntará los resultados de las pruebas, su justificación y el incentivo o impedimento conseguido si lo hubiere.

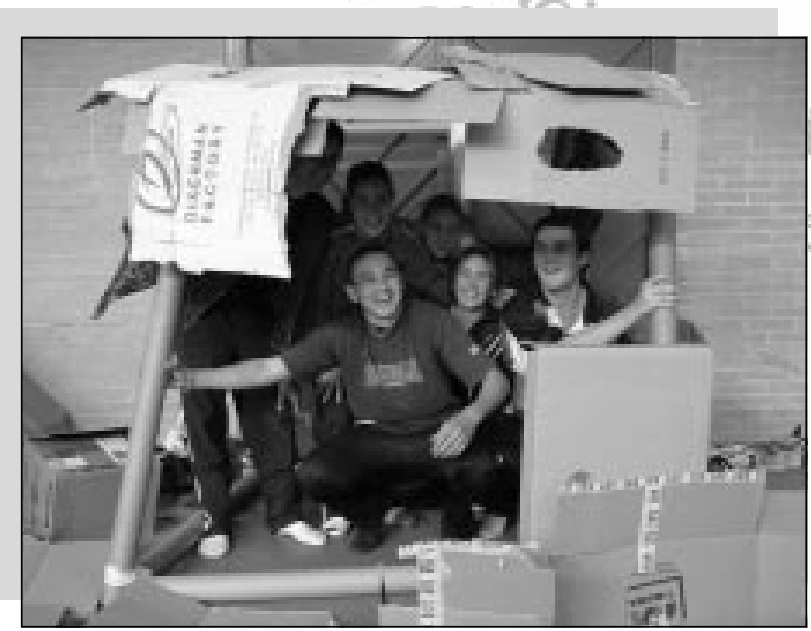

Esta tabla o planilla debe estar visible a todos los grupos y al dinamizador, ya que de esta manera se pueden ir comparando las distintas soluciones que se dan a una misma situación.

Al finalizar el juego se utilizará para realizar la reflexión final, pudiendo ver el desarrollo del proceso del juego de cada uno de los grupos (ver tabla 1).

\section{Una experiencia prácica en secundaria}

La experiencia que a continuación se detalla se realizó en el Instituto de secundaria de Benicasim con los cuatro grupos de $4^{\circ}$ de ESO como motivo de la presentación del viaje de multiaventuras que iban a realizar. Un total de 90 alumnos disfrutaron de esta práctica de tiempo libre con gran aceptación.

Una vez concentrados en el pabellón del Instituto se empezó con un pequeño calentamiento recreativo llamado "Fleix" que mezcla distensión articular con ritmo y canto.

La agrupación en pequeños grupos la realizaron los propios alumnos, dejando un margen de entre cuatro y seis el número de componentes

Rápidamente se les pidió que se quitaran las zapatillas, todos los utensilios de los bolsillos y cazadoras, jerséis y otras prendas innecesarias para la práctica. Tras el asombro de los participantes se pasó a ponerlos en situación:

iEn estos momentos sois supervivientes de una catástrofe y solo contáis con vosotros mismos y con el siguiente equipo de supervivencia: 10 metros de cuerda, unas tijeras, 4 bolsas de basura, 2 metros cuadrados de tela, una botella de agua agujereada, tres cajas de cartón, un bolígrafo, y un rollo de precinto. Esto es todo, vuestro ob-

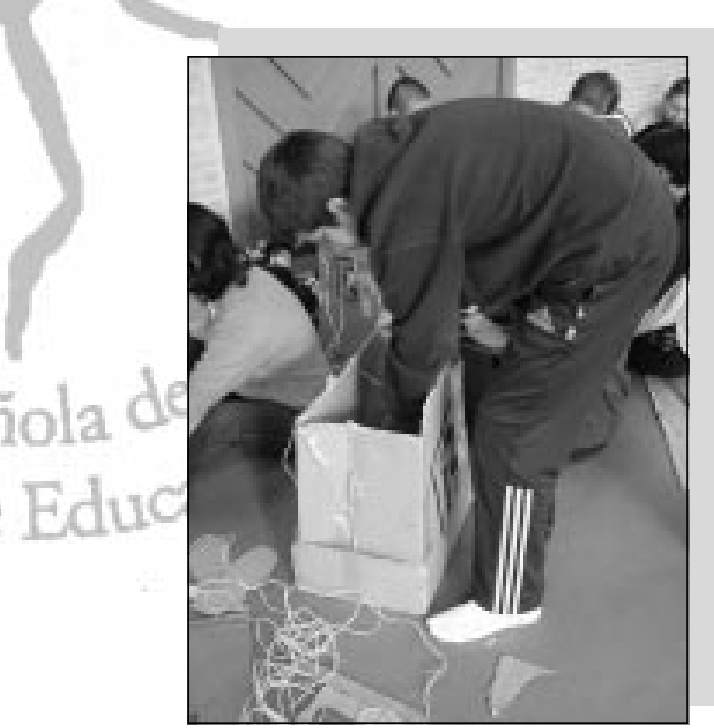

jetivo es superar las diferentes pruebas que las circunstancias de la catástrofe generen. De vuestra destreza y economía en la utilización del material, así como de vuestro trabajo en equipo, depende que sobreviváis. Suerte!

La primera prueba a realizar fue la de confección de zapatos de circunstancia y de la mochila para llevar todo el equipo de supervivencia. Tenían 15 minutos, una vez pasado el tiempo, todo aquel y aquella que no tuviera zapatos se quedaría ciego de un ojo para el resto del juego. Fueron varias las opciones y modelos de zapatos que aparecieron en los diferentes grupos, algunos de ellos muy originales pero poco útiles, otros muy buenos pero demasiado material utilizado...

La mochila para el material de supervivencia debía cumplir el requisito de albergar todo el material y tenia que ir a la espalda por lo que los diseños estuvieron condicionados a las características pedidas. A partir de una caja, con la bolsa de basura, algunos de ellos llegaron a coser la tela con la cuerda para hacer una especie de bolsa, con el inconveniente que no pudieron reciclar el material utilizando condicionando el desarrollo de las siguientes pruebas. 


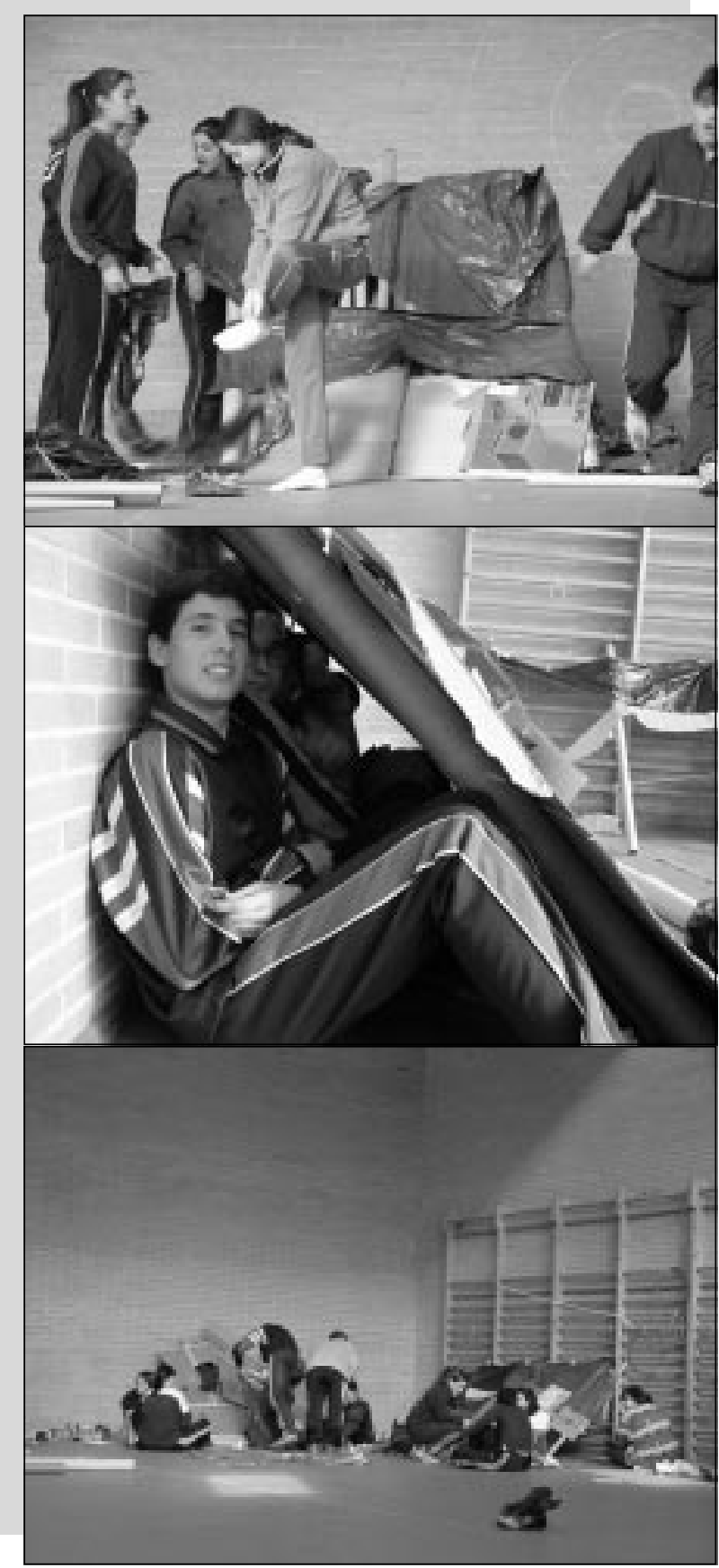

iLa noche se acerca con la previsión de una fuerte tormenta, por lo tanto tenéis que construir un refugio donde quepa todo el grupo, que sea resistente al viento y sobre todo que cubra del agua, tenéis 30 minutos. Desde este preciso instante. Manos a la Obra!. Esta fue la siguiente consigna y los resultados:

Esta vez si que hubieron penalizaciones porque algunas de las cabañas no cumplían los tres requisitos: resistente, capacidad para todo el grupo y que cubra de la lluvia. En fin, todo grupo que su refugio no cumplía los requisitos debían atarse por parejas de la muñeca de una de las dos manos (lo que provocaba mayor cooperación y coordinación del grupo para realizar la siguiente prueba).

Las cosas se iban complicando para algunos, la siguiente prueba se trataba de realizar un rastreo por el patio. Iban a tener la oportunidad de comprobar la utilidad práctica del calzado, así como, las ventajas e inconvenientes del modelo diseñado. En esta prueba se les facilitó una planilla el significado de las diferentes pistas que iban a
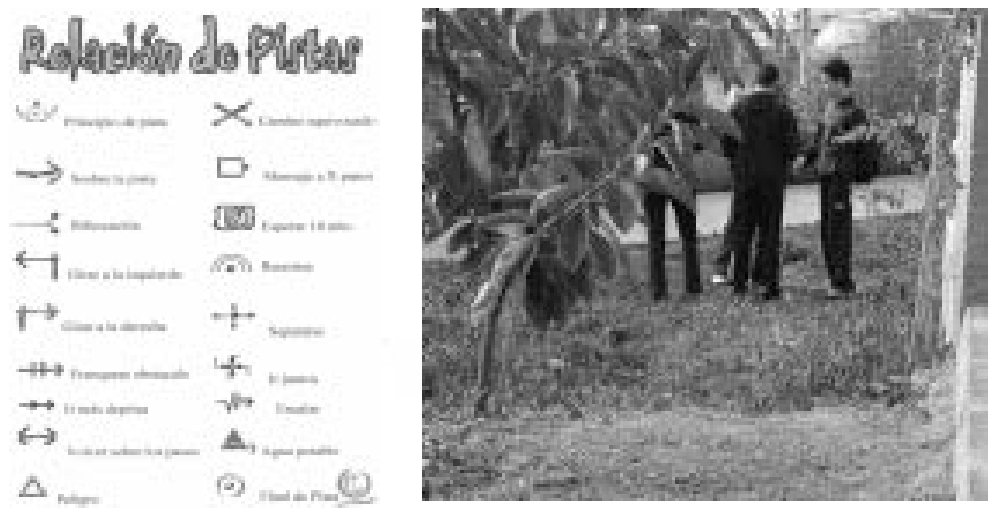

encontrar a lo largo del recorrido. El objetivo de la prueba era dibujar el recorrido y realizar las diferentes pruebas que se explicaban en algunas de las pistas.

Llegado a este punto el material empezaba a escasear y todavía estábamos a mitad del juego. La siguiente prueba era de transporte de agua. Tenían una botella de agua llena y tenían que realizar un recorrido para llegar a otra botella y llenarla. No podían utilizar la boca y solo se les permitía perder un $10 \%$ del agua que tenían. Todo un reto de ingeniería para diseñar un sistema de transporte de agua. Nadie superó la prueba con éxito debido a su complejidad pero la búsqueda, por la impermeabilidad que permitiría llevar el agua, fue muy interesante, desde el plástico al papel, desde las propias manos hasta cacharros de cartón. En esta prueba empezaron a aparecer la necesidad de reciclar material ya utilizado como el precinto de los zapatos y alguna que otra bolsa de basura que cubría el refugio así como los tubos de cartón.

\section{Nuestros objetivos se estaban cumpliendo}

iEstimados participantes la siguiente prueba viene explicada en este papel, el código utilizado es el conocido morse. Esperemos que lo conozcáis, para todos aquellos que no lo conozcáis existe una planilla que podéis adquirir mediante el cambio con material del equipo de supervivencia, si descifráis el mensaje antes del tiempo establecido (15 minutos a partir de este momento) no sólo recuperáis el material, si no que se os dará la oportunidad de elegir tres materiales de una lista. Seguro que podéis hacerlo! Esta consigna encabezaba una hoja en la que había un mensaje en morse donde se les pedía que representaran el cuento de caperucita roja a los dinamizadores.

La última prueba hacia referencia a la escalada. Se construyó un rocódromo en las espalderas de la instala-

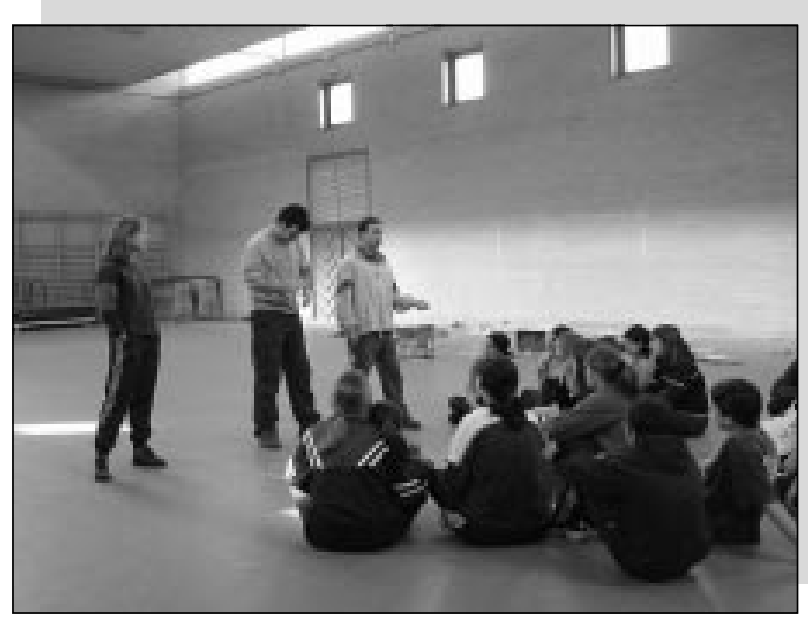



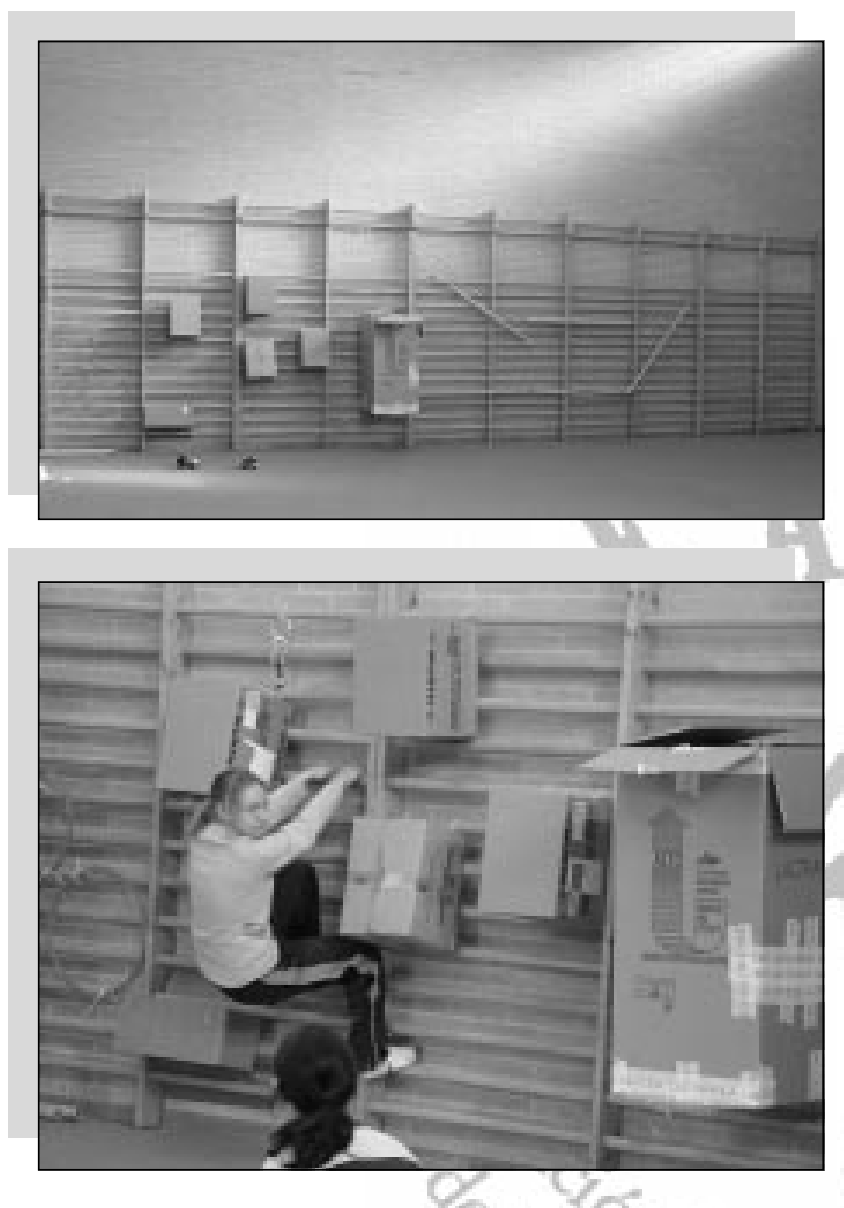

ción con cajas de cartón, cuerdas, aros y bridas, donde los diferentes grupos debían colocar las cintas de escalada en los lugares señalados. Una prueba en apariencia fácil pero de máxima complejidad debido a la dificultad añadida de los zapatos de circunstancia (que se resbalaban) y los diferentes impedimentos que habían ido acumulando a lo largo del juego.

Finalmente y cerrando la actividad se realizó una reflexión en grupo donde se destacaron algunos aspectos positivos de la experiencia:

- iLo hemos pasado muy bien!

- iNunca pensé que saliera al patio con unas zapatillas fabricadas por mi!

- icon tan poco material hemos hecho tantas cosas..!

- ¿ya se ha terminado?

Por otro lado los dinamizadores resaltaron la participación activa del grupo en general y sobre todo el aspecto ecológico de actividad y como para pasarlo bien no es necesario gran cosa.

\section{"Supervivencia Urbana" algo más que un juego}

Una vez más nos preguntamos si verdaderamente se puede educar a través de actividades recreativas, o si éstas sólo nos ayudan a pasar un rato divertido y saber más juegos y actividades para poder realizar con mis amigos, con mis hijos, con mi equipo durante una concentración...
En el proceso de desarrollo del juego (gráfico 1) observamos que los términos utilizados corresponden a la planificación y dinamización de actividades recreativas y de tiempo libre. En él aparecen palabras como DINAMIZADOR, PARTICIPANTES, PRUEBAS, PLANILLA DE RESULTADOS, palabras que podemos reemplazar por MAESTR@ o PROFES@R, ALUMN@S, TAREAS o ACTIVIDADES, TABLA DE EVALUACIÓN.

En este momento estamos trasladando el juego a una clase de Educación Física, un juego durante el cuál se nos plantea una continua resolución de problemas que los alumnos/as deben superar. Estos problemas no son más que actividades diseñadas por el profesor/a o maestro/a, con contenidos elegidos por ellos mismos para conseguir los objetivos propuestos.

Estamos pues ante una actividad educativa, enmarcada dentro de un estilo de enseñanza que llamaremos de resolución de problemas continuos, estilo que en el espectro que nos describe Mosston y Ashworth (1993), se encuentra situado dentro de los estilos que desarrollan al máximo los canales tanto físicos como cognitivos.

Por eso este estilo en el espectro proporciona a los alumnos una oportunidad legítima para examinar ideas divergentes y descubrir alternativas. La palabra independencia toma aquí un cierto protagonismo. Además tanto el canal emocional como el social los alumnos se sitúan en el máximo ya que los procesos de descubrimiento se realizan con compañeros.

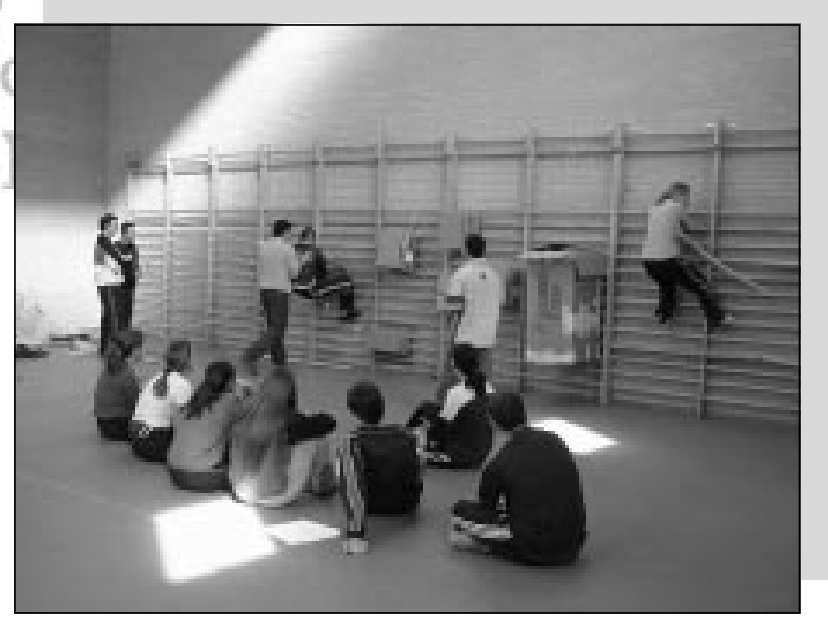

Es por todo lo anterior que los contenidos que la recreación nos proporciona, no son inherentes al proceso educativo de los alumnos/as sino que además en la mayoría de casos desarrollan al máximo los canales tanto físicos como cognitivos y sobre todo sociales.

Así la "Supervivencia Urbana" no sólo es una forma de divertirse sino un proceso educativo a través del cual estamos ayudando a los participantes o alumn@s, a despertar su creatividad, su cooperación, su afán de superación, su interés por el descubrimiento, y sobre todo y con la ayuda de actividades que normalmente son propias de la naturaleza, transmitir una serie de valores, cada vez más perdidos en los distintos entornos urbanos en las que vivimos, de entre los cuales la igualdad, la ecología, el consumismo moderado y la cooperación son necesarios para transformar la sociedad en la que vivimos. 


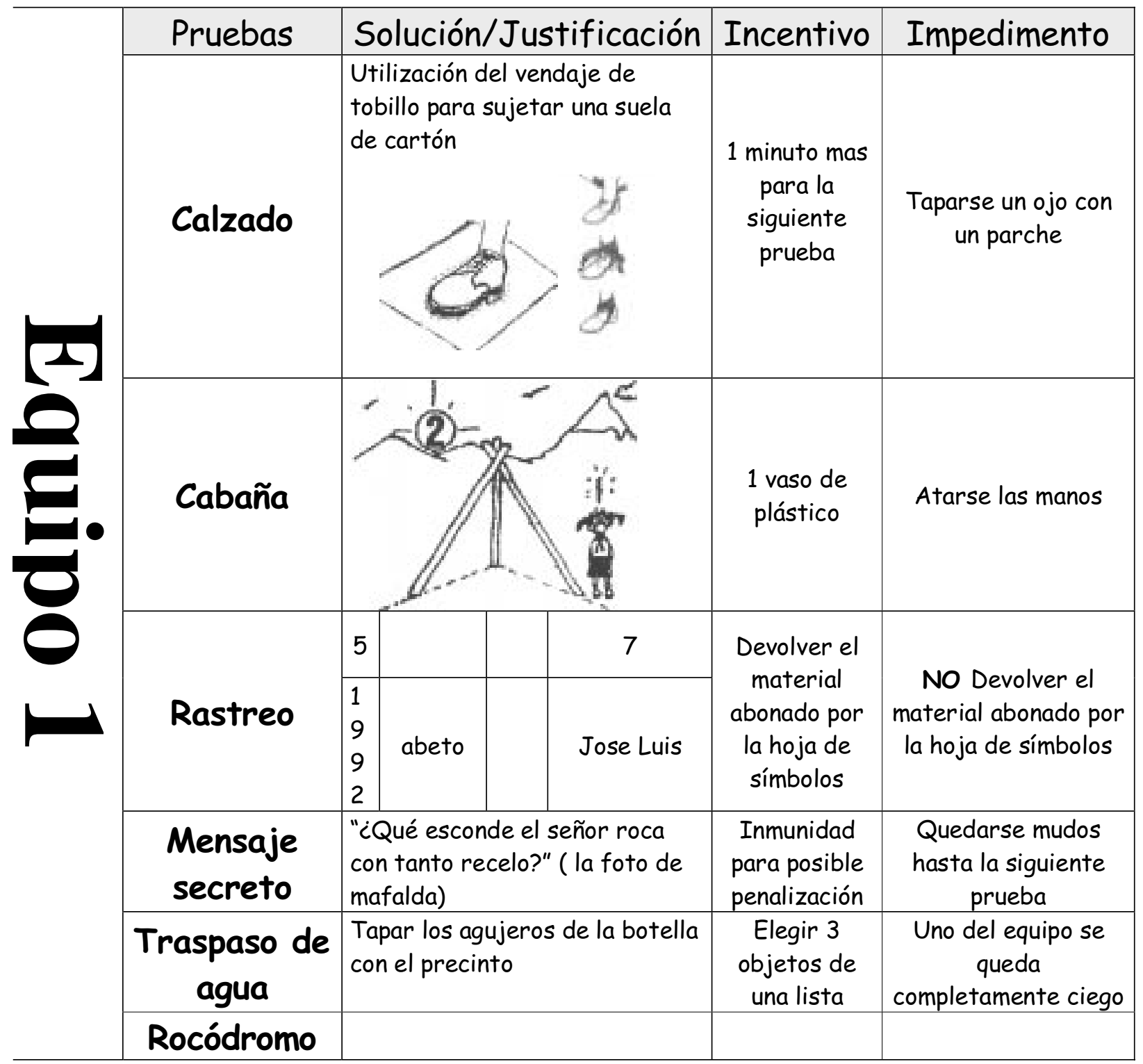

Tabla 1.

\section{Bibliografía}

Camerino, O. (2000). Deporte Recreativo. Biblioteca Temática del Deporte. INDE.

García Montes, M. E. (1992). Descubrir la ciudad como un espacio de recreación. El deporte de orientación como juego en el medio urbano. En Simposi internacional de filosofia de l'esport. Educació ètica iestètica de l'activitat física i l'esport, 121-124. Barcelona: Universitat Autónoma de Barcelona.

García Montes, M. E. y Hernández Rodríguez, I. (1998). Recorridos de orientación urbana: una forma de apropiarse de la ciudad. Revista Espacio y Tiempo, 22-23-24, 5569.

Jardi, C. y Pinyol, J. (1990). 1000 ejercicios y juegos con material alternativo. Barcelona: Paidotribo. Colección deporte.

López Quintas, A. (2000). Tiempo de ocio, tiempo de creatividad. En M. Cuenca Cabeza (ed.): Ocio y desarrollo humano. Propuestas para el $6^{\circ}$ Congreso Mundial de Ocio, 85-91, Bilbao. Universidad de Deusto.
Mosston, M. y Ashworth, S. (1993). La Enseñanza de la Educación Física. Hispano Europea.

Nehberg, R. (1987). Manual del Aventurero. Técnicas de supervivencia. Martínez Roca.

Olivera, J. y Olivera, A. (1995). La crisis de la modernidad y el advenimiento de la posmodernidad: el deporte y las prácticas físicas alternativas en el tiempo de ocio activo. Apunts, Educación física y deportes, 41, 10-29.

Posada, F. (2001). Transversalidad y educación en valores en Educación Física. Revista de Educación Física, 74, 15-24.

Ruiz Juan, F.; García Montes, M. E. y Hernández Rodríguez, A. I. (2000). Organización de jornadas lúdicas en espacios no convencionales. Almería: Universidad de Almería.

Ruiz Juan, F.; García Montes, M. E. y Hernández Rodríguez, A. I. (2001).El interés por la práctica de actividades físico-deportivas de tiempo libre del alumnado de la Universidad de Almería. Un estudio longitudinal. Apunts. Educación Física y Deportes, 63, 86-92.

Ruiz Juan, F.; García Montes, M. E. (2001). Desarrollo de la Motricidad a través del Juego. Materiales convencionales y no convencionales como recursos metodológicos. Madrid: Gymnos. 